\title{
The NIMH Multisite HIV Prevention Trial: Reducing HIV Sexual Risk Behavior
}

\section{The National Institute of Mental Health (NIMH) Multisite HIV Prevention Trial Group*}

The efficacy of a behavioral intervention to reduce human immunodeficiency virus (HIV) risk behaviors was tested in a randomized, controlled trial with three high-risk populations at 37 clinics from seven sites across the United States. Compared with the 1855 individuals in the control condition, the 1851 participants assigned to a small-group, seven-session HIV risk reduction program reported fewer unprotected sexual acts, had higher levels of condom use, and were more likely to use condoms consistently over a 12-month follow-up period. On the basis of clinical record review, no difference in overall sexually transmitted disease (STD) reinfection rate was found between intervention and control condition participants. However, among men recruited from STD clinics, those assigned to the intervention condition had a gonorrhea incidence rate one-half that of those in the control condition. Intervention condition participants also reported fewer STD symptoms over the 12-month follow-up period. Study outcomes suggest that behavioral interventions can reduce HIV-related sexual risk behavior among low-income women and men served in public health settings. Studies that test strategies for reducing sexual risk behavior over longer periods of time are needed, especially with populations that remain most vulnerable to HIV infection.

The Public Health Service has identified HIV infection as the most critical present threat to the nation's public health. Rates of HIV infection and acquired immune deficiency syndrome (AIDS) are higher among racial and ethnic minority populations, especially African Americans, who represented $41 \%$ of new AIDS cases in 1996. Several trends in the epidemic are especially disturbing: People are most affected during their productive years, infection appears to be occurring at increasingly younger ages, and heterosexual transmission is rising rapidly, especially among ethnic minority women (1). These trends underscore the need for interventions that effectively reduce sexual risk behavior among ethnic minority heterosexual adults living in high HIV seroprevalence areas.

In response to directives from Congress, the National Institute of Mental Health (NIMH) initiated the NIMH Multisite HIV Prevention Trial in 1990 to develop a risk reduction intervention based on current best practices in HIV prevention and to test its efficacy with understudied and disadvantaged populations at multiple sites across the United States. This article describes the main findings of the trial, a study that included both self-reported and biological outcomes and a common protocol at

${ }^{*}$ Correspondence should be addressed to Multisite Staft Collaborator, National Institute of Mental Health, National Institutes of Health, Parklawn Building, Room 18-101, 5600 Fishers Lane, Rockville, MD 20857, USA. multiple sites (2).

The trial was implemented in 37 innercity, community-based clinics located in five metropolitan areas. NIMH funded principal investigators in seven sites: the Bronx and Harlem, New York City; Manhattan and Brooklyn, New York City, and northern New Jersey; Baltimore, Maryland; Atlanta, Georgia; Milwaukee, Wisconsin; Los Angeles, California; and Orange and San Bernardino counties, California. Participants were recruited between January 1994 and February 1996 from three distinct populations at risk of acquiring HIV and other sexually transmitted diseases (STDs): men and women in STD clinics and women attending health service organizations (HSOs), mostly primary care clinics (3).

Recruitment procedures. Participants were screened for HIV-related high-risk acts with a reliable and sensitive procedure (4) in the waiting rooms of all recruitment clinics. Eligibility criteria included the following: age (20 years or older for STD participants, 18 years or older for HSO women), engaging in unprotected vaginal or anal sex in the past 90 days, and having at least one of the following over the past 90 days: sex with a new sexual partner, more than one sexual partner, an STD, a sexual partner they knew also had other sexual partners, or sex with an injection drug user or a person infected with HIV. Most persons reported primarily heterosex- ual behavior, although $7 \%$ of male participants reported having sex with men during the 3 months before the baseline interview. Of 38,893 persons screened, 3706 participants were randomized, representing $33 \%$ of those eligible at screening. Of these, 1564 men and 862 women were recruited from STD clinics and 1280 women were recruited from HSOs.

Statistical power. To determine the study's sample sizes, power analyses were conducted on the basis of two dichotomous end points: consistent condom use and STD reinfection rate from chart abstraction data for male participants recruited through STD clinics. A goal of $90 \%$ power was chosen to detect differences at the $\alpha=0.05$ level (two-tailed test) between the intervention and control groups within each population (that is, STD males, STD females, and female participants in $\mathrm{HSOs}$ ) at 12 months of follow-up. Sample sizes were computed to detect a $15 \%$ difference in consistent condom use and a 10\% difference in STD infection rate. It was further assumed that $95 \%$ of subjects randomized would be at risk and that $72 \%$ of these participants would complete the 12 -month follow-up interview.

Assessment interviews. All participants were assessed at baseline and again at 3, 6, and 12 months after the intervention. The baseline interview assessed sociodemographic characteristics, physical and mental health status, STD symptoms, alcohol and drug use, sexual behavior, and HIV testing. Assessment interviewers were blind to intervention condition and did not serve as group facilitators. Moreover, quality control procedures were rigorously implemented to ensure accuracy of reported data (2). Completion rates for follow-up interviews were $82 \%$ or greater for participants in both the intervention and control conditions across all follow-up assessment points.

Experimental conditions. Participants assigned to the control condition attended a 1-hour AIDS education session that included a videotape (5) and a question-andanswer period.

Participants assigned to the intervention condition were asked to attend seven 90- to 120-min HIV risk reduction sessions, conducted twice weekly in groups of 5 to 15 persons (2). Groups were composed separately of male or female participants and were usually co-led by a male and a female facilitator with previous group experience who received extensive training and were certified before initiating group sessions. Fidelity to the intervention protocol was monitored through periodic site visits and systematic review of audiotapes of all sessions and videotapes of a random subset of sessions Participants assigned to the inter- 
vention condition attended an average of 5.2 of the seven sessions $(\mathrm{SD}=2.0)$, and $63 \%$ of participants attended six or all seven sessions

End points. Primary end points for the trial were (i) self-reported number of unprotected vaginal and anal intercourse acts during the 90 days before each interview, (ii) self-reported consistent condom use during the 90 days before each interview, (iii) the proportion of vaginal or anal intercourse acts in which a condom was used during the 90 days before each interview (self-reported), (iv) STD reinfection rate from chart review during the period from the end of the intervention through the 12-month interview window (male STD participants only), and (v) point prevalence of chlamydia and gonorrhea at the 12month assessment with DNA amplification [ligase chain reaction (LCR)] of urine specimens. Secondary end points included (i) participant self-reports of STD symptoms during the 90 days before each interview and (ii) incidence of gonorrhea during the follow-up period from chart review for STD participants.

Participants were asked at each interview to indicate the number of people with whom they had sex during the past 90 days.

Table 1. Sociodemographic and risk behavior characteristics for persons eligible at baseline and participants randomized in the study.

\begin{tabular}{|c|c|c|}
\hline & \multicolumn{2}{|c|}{ Randomized (\%) } \\
\hline & Control & Intervention \\
\hline \multicolumn{3}{|c|}{ Sociodemographics } \\
\hline Male & 42.1 & 42.3 \\
\hline$<25$ years of age & 24.8 & 25.2 \\
\hline Hispanic & 25.6 & 25.2 \\
\hline African American* & 73.9 & 74.0 \\
\hline$\geq$ High school & 54.8 & 55.5 \\
\hline Never married & 61.7 & 62.3 \\
\hline Employed & 29.0 & 30.6 \\
\hline \multicolumn{3}{|c|}{ Risks } \\
\hline (90 days) & 61.6 & 59.4 \\
\hline $\begin{array}{c}3+\text { partners } \\
\text { (90 days) }\end{array}$ & 350 & 342 \\
\hline Ever HIV tested & 82.8 & 82.8 \\
\hline$\geq 11$ risky acts & & \\
\hline $\begin{array}{l}\text { (90 days) } \\
\text { 0\% condom use }\end{array}$ & 53.1 & 51.9 \\
\hline $\begin{array}{l}\text { (90 days) } \\
0 \text { to } 50 \% \text { condom use }\end{array}$ & 44.7 & 44.2 \\
\hline (90 days) & $83.7 \dagger$ & 80.4 \\
\hline Drug use & & \\
\hline $\begin{array}{c}\text { (90 days) } \\
\text { Injected drugs }\end{array}$ & 53.5 & 53.2 \\
\hline (90 days) & 6.7 & 6.9 \\
\hline Ever had STD & 73.1 & 73.0 \\
\hline Sample size & 1855 & 1851 \\
\hline
\end{tabular}

*Includes African Americans of Hispanic descent. $\dagger$ Indicates a significant difference between the control and intervention conditions at the $P<0.05$ level, with a $x^{2}$ test of association.
The total number of vaginal or anal intercourse acts (or both) and the total number of times a condom was used were computed for each participant by summing events across all partners. From this information, the three indices of sexual risk were constructed (2).

Statistical methods. Behavioral end points (number of unprotected vaginal or anal intercourse acts, proportion of intercourse acts protected by condoms, and consistent condom use or abstinence) were analyzed for intervention effects across follow-up assessments and by population. All models for the number of unprotected intercourse acts were fit to the square root of the measure. For all analyses, basic model effects included study population (overall model only), baseline value of the outcome measure (except consistent condom use or abstinence for which all baseline values were zero, because of study eligibility requirements), study site, and intervention assignment.

Longitudinal analyses were conducted by fitting linear repeated measures models or repeated measures logistic regression models across follow-up assessments with generalized estimating equation (GEE) methodology (6). Participants reporting no sexual activity during the previous 90 days at a given follow-up assessment (typically 10 to $12 \%$ ) were assigned values corresponding to consistent condom use for all behavioral outcomes. Separate models were fit to the data for the total sample and for each study population. Effects for time and time by experimental condition interactions were added to the basic model. Tests of intervention effects at each of the three follow-up assessment points and overall, as well as tests of time by intervention condition interactions, were computed by Wald $\chi^{2}$ tests.

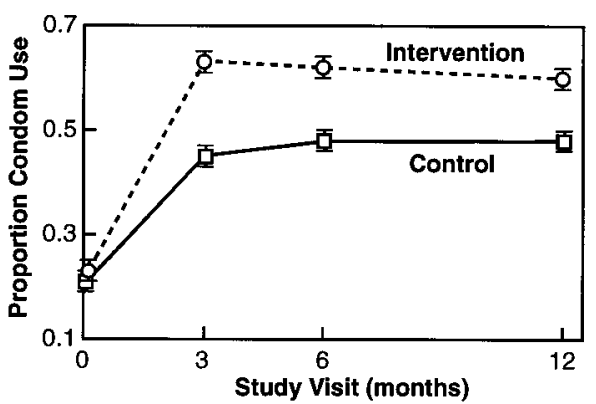

Fig. 1. Mean proportion of condom use overall at baseline and at 3-, 6-, and 12-month follow-ups by intervention assignment. Brackets at each time point show 95\% confidence limits on the mean. Means increased from 0.21 to 0.23 at baseline to about 0.60 for the intervention condition during follow-up and 0.45 to 0.48 for the control condition. The effect size was 0.47 at 3 months, 0.36 at 6 months, and 0.31 at 12 months.
Analysis of covariance (ANCOVA) models were fit to each behavioral outcome measured at the 12-month assessment to assess whether the intervention effect remained when missing 12-month outcome values were imputed, with three imputation methods: (i) a last observation forward approach for all participants who completed the baseline and at least one follow-up assessment, (ii) a method (7) based on response within strata defined by outcomes or nonresponse at 3- and 6-month follow-ups, and (iii) a method that uses baseline values to replace missing data at the 12 -month assessments.

The contribution of intervention condition attendance to the outcome effects was examined by fitting ANCOVA models to each 12-month follow-up outcome with a three-level attendance indicator (control, attended five or fewer sessions, or attended six or seven sessions).

Differential intervention effects across subgroups defined by participant characteristics were assessed by including baseline covariate effects and intervention condition by covariate interactions in ANCOVA models fit to each outcome measured at the 12-month follow-up assessment.

Self-reports were used to determine the presence of STD-related symptoms during follow-up. For participants recruited in STD clinics, indicators of incident STDs both overall and by type (gonorrhea, chlamydia, syphilis, trichomoniasis, and nongonoccocal urethritis) at any time during the 12month follow-up period were created from chart abstraction data. Dichotomous indicators of gonorrhea and chlamydia were also created on the basis of urine tests completed at the 12-month follow-up assessment. For each of these indicators, the intervention and control condition results were compared by Cochran-Mantel-Haenszel $\chi^{2}$ tests stratified by study population (overall test only) and study site.

Who participated in the trial? The large majority of participants were African American or Hispanic; most were single, unemployed, and treated previously for STDs, and all were at high behavioral risk for HIV (Table 1). The sociodemographic and sexual behavior characteristics of control and intervention condition participants were very similar. The two groups differed slightly in condom use rate at baseline; a slightly higher percentage $(83.7 \%)$ of control condition participants than intervention condition participants $(80.4 \%)$ reported that they used condoms half or less of the time. Of those tested for HIV, 54\% disclosed the results of the test (8).

Did the intervention produce changes in sexual behavior? Reported frequency of unprotected intercourse acts decreased in 
the follow-up period for participants in both study conditions. Participants in the intervention condition reduced their frequency of unprotected intercourse acts significantly more than control condition participants at each follow-up assessment $(P<0.0001$ across all follow-up points; Table 2). Mean frequency of unprotected intercourse acts among intervention condition participants declined by about $50 \%$ from baseline levels to 12.9 acts at 3 months, 12.5 acts at 6 months, and 12.0 acts at the 12 -month follow-up, reflecting intervention effect sizes of -0.29 at the 3 -month follow-up and -0.21 at the 12 -month follow-up. Longitudinal analyses also indicated a significant overall decrease in unprotected intercourse acts for the intervention condition. Very similar patterns of change were found across the three study populations.

Although levels of condom use increased over time among control condition participants, the increase in condom use by participants who received the intervention was significantly greater $(P<0.0001$ across all follow-up points; Table 3 and Fig. 1). Among intervention condition members overall, only $23 \%$ of intercourse acts in the 90 days preceding the baseline assessment were protected by condoms. Condom use increased to $63 \%$ of all intercourse acts at 3-month follow-up and never declined below $60 \%$ in any subsequent follow-up, reflecting effect sizes of 0.47 at the 3-month follow-up and 0.31 at the 12-month followup. Longitudinal analyses again showed significantly greater condom use overall among the intervention condition participants. Similar patterns were evident in all three of the study populations.

At baseline, enrollment criteria ensured that no study participants were consistent condom users (or sexually abstinent) in the previous 90 days. At the 3-month followup, $42.0 \%$ of intervention condition participants were consistently using condoms or were abstinent; consistent condom use or abstinence was reported by $44.1 \%$ of intervention condition members at the 6-month follow-up and $43.4 \%$ at the 12 -month follow-up. Although there was also an increase
Table 2. Number of unprotected intercourse acts in the 90 days before baseline and at 3-, 6-, and 12-month follow-ups by intervention type and population. The table is based on all nonmissing visits at each time point. All participants included in this table and Tables 3 and 4 had to have at least one follow-up interview. Because $90 \%$ of the participants completed one or more follow-up interviews, the sample size for the baseline results was about $90 \%$ of that of the full sample. Assessments occurring outside of allowable windows for each follow-up period
(4 to 5\%) were excluded from behavioral outcome analyses, with windows defined as follows: 2 to 4.5 months for 3-month follow-up assessments, 4.5 to 8 months for 6-month assessments, and 10.5 to 15 months for 12 -month assessments. Additionally, a very few participants at each time point were unable to specify either the number of times they had intercourse or the number of times they used a condom or both, so the behavioral outcomes could not be calculated for that visit. C, control; I, intervention.

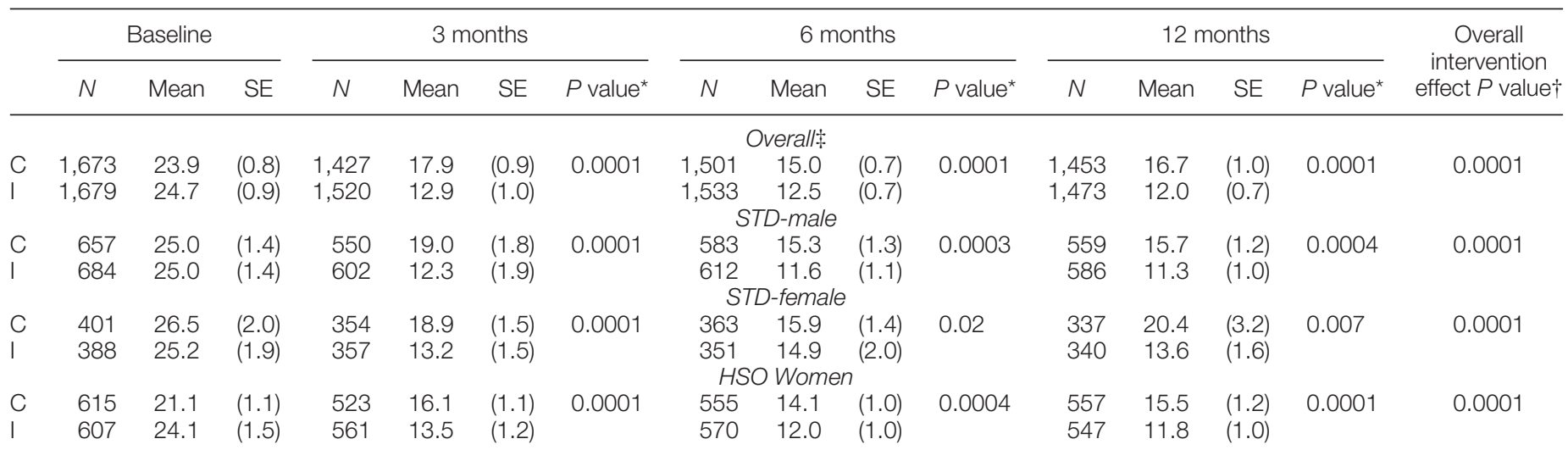

${ }^{*} P$ value for a test of intervention compared with control at the specified follow-up visit from longitudinal models fit to square-root transformed outcome. Separate models were fit to the data overall and for each study population and included effects for baseline value of the end point, intervention assignment, site, study population (total only), time, and time by intervention assignment interaction. $\quad \uparrow P$ value for an overall test of intervention compared with control. Longitudinal models were fit as above excluding time by intervention assignment interaction. \$Over the three study populations.

Table 3. Proportion of condom use in the 90 days before baseline and at 3-, 6-, and 12-month follow-ups by intervention type and population. See Table 2 legend. C, control; I, intervention.

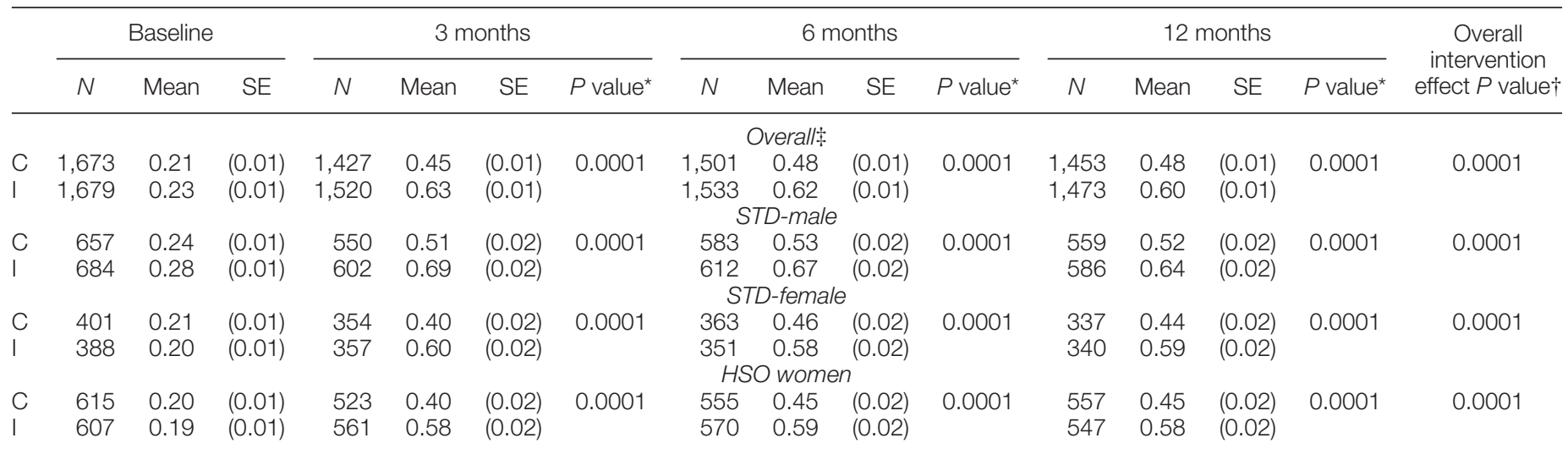

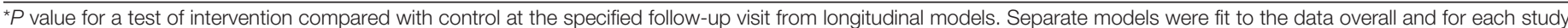

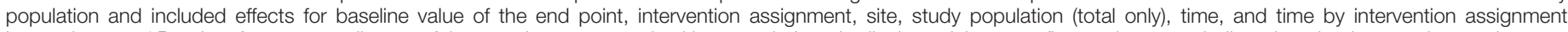

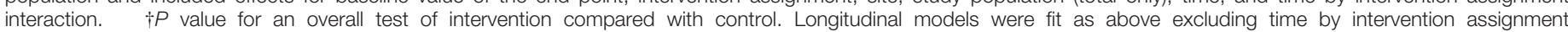
interaction. $\quad$ \$Over the three study populations. 
in consistent condom use among control condition members (that is, about one-third of control participants reported consistent condom use at the 12-month follow-up), the magnitude of change was significantly greater among intervention condition participants $(P<0.0001$ at each follow-up point and longitudinally; Table 4). The three populations showed similar patterns. For all behavioral outcomes, the same pattern of results emerged when missing values were imputed (9)

Participants who attended more intervention sessions exhibited greater magnitudes of behavior change. A significant intervention by dose (number of sessions attended) interaction was observed for number of unprotected intercourse acts, proportion of condom use, and consistent condom use or abstinence. For example, the mean number of unprotected intercourse acts at the 12-month follow-up was 16.7 acts for participants in the control condition, compared with 13.2 acts for intervention condition participants who had attended five or fewer sessions $(P=0.03)$ and 11.2 acts for those who had attended six or all seven sessions $(P<0.0001)$.

ANCOVA was used to assess the consistency of the intervention effect across subgroups defined by baseline characteristics. Differential effects, with respect to the three behavioral outcome measures at the 12-month follow-up, were not observed across subgroups defined by age, education, race or ethnicity, baseline alcohol abuse symptoms, drug use (past 90 days), commercial or survival sex (past 90 days), unwanted sexual activity, or mental health service use (past 90 days).

Did the intervention produce change in rates of incident STDs? About one in three $(34.6 \%)$ control condition members reported experiencing symptoms indicative of an STD at one or more follow-up points, significantly higher than the rate of $27.9 \%$ among intervention condition members $(P=0.001$; Table 5$)$. This significant pattern was found consistently across the three study populations.

Overall, about $9 \%$ of the participants recruited from STD clinics evidenced an incident STD on the basis of clinic chart review (Table 5). Although no difference was observed between intervention or control conditions, aggregating data may mask outcome effects because, in the United States, incidence of sexually transmitted disease differs by age and gender (10). Gonorrhea is more prevalent among men than women over the age of 24 years, and it is generally more symptomatic in men than women (11). Additionally, gonorrhea is easily detected by virtually all public health clinics (12). Thus, incident gonorrhea was examined separately among male participants. Chart records indicated that $6.4 \%$ of STD clinic control condition men were treated for incident gonorrhea in the follow-up year compared with only $3.6 \%$ among intervention condition STD clinic men $(P<0.03)$

The LCR urinalysis at 12-month followup revealed point prevalence for gonorrhea of $1.5 \%$ among control condition members and $0.9 \%$ among intervention condition members. Although in a direction consistent with the other measures of gonorrhea follow-up incidence and consistent across

Table 5. Results of symptoms, clinical chart review (diagnosed with at least one STD), and urine specimen analysis by population and intervention type. Table gives percentage of participants. C, control; I, intervention.

\begin{tabular}{|c|c|c|c|c|c|c|c|c|}
\hline & \multicolumn{2}{|c|}{ Symptoms* } & \multicolumn{3}{|c|}{ Clinical chart review } & \multicolumn{3}{|c|}{ Urine specimen } \\
\hline & $N$ & Percentage & $N$ & All STDs† (\%) & GC (\%) & $\mathrm{N}$ & CT (\%) & GC (\%) \\
\hline \multicolumn{9}{|c|}{ Overall } \\
\hline C & 1,729 & $34.6 \$$ & 904 & 9.4 & $5.0 \%$ & 1,296 & 2.8 & 1.5 \\
\hline | & 1,722 & 27.9 & 922 & $\begin{array}{l}9.1 \\
\text { STD-male }\end{array}$ & 3.0 & 1,285 & 2.9 & 0.9 \\
\hline C & 692 & $17.5 \neq$ & 576 & 9.9 & $6.4 \ddagger$ & 499 & 1.8 & 2.0 \\
\hline | & 706 & 12.5 & 583 & $\begin{array}{c}8.2 \\
\text { STD-female }\end{array}$ & 3.6 & 505 & 3.6 & 1.0 \\
\hline C & 413 & $47.9 \%$ & 328 & 8.5 & 2.4 & 313 & 1.9 & 1.9 \\
\hline । & 400 & 41.0 & 339 & $\begin{array}{c}10.6 \\
\text { HSO women }\end{array}$ & 2.1 & 288 & 2.1 & 1.4 \\
\hline C & 624 & $44.7 \ddagger$ & $-\S$ & - & - & 484 & 4.3 & 0.8 \\
\hline । & 616 & 37.0 & - & - & - & 492 & 2.6 & 0.6 \\
\hline
\end{tabular}

${ }^{*}$ At any follow-up. †Gonorrhea (GC), chlamydia (CT), nongonoccocal urethritis (for males only), syphilis, and trichomoniasis. ¥Control and intervention significantly different at 0.05 level. \$No biological abstraction was done for women recruited from HSOs.

Table 4. Percentage of consistent condom use or abstinence in the 90 days before baseline and at 3-, 6-, and 12-month follow-ups by intervention type and

ipants reporting consistent condom use at baseline were ineligible for the trial; thus, the percentage of participants reporting consistent condom use is zero at population. The table is based on all nonmissing visits at each time point. Particbaseline by definition. Also see Table 2 legend. C, control; I, intervention.

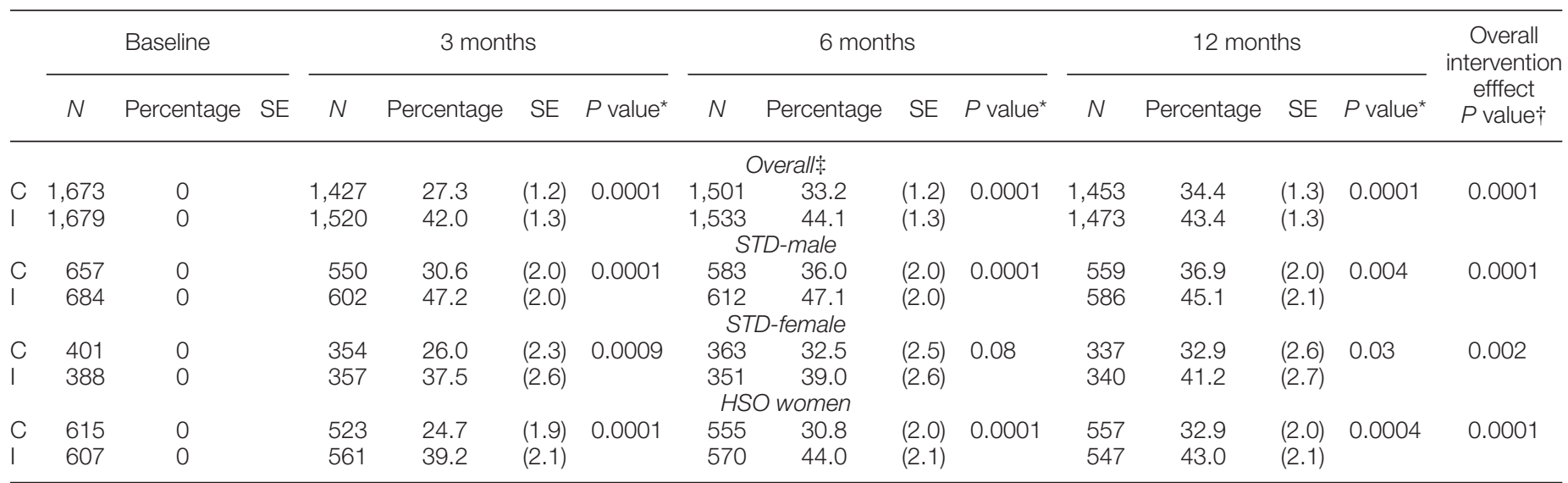

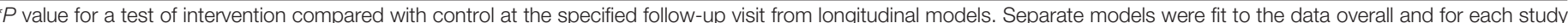

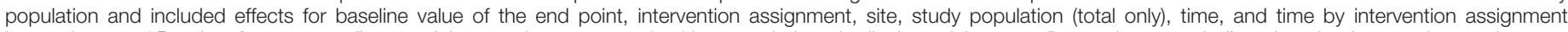

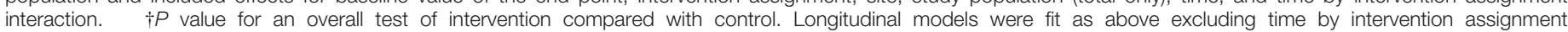
interaction. $\quad$ \$Over the three study populations. 
the three study populations, this difference was not statistically significant.

Discussion. Relative to participants in a control condition who received only a single-session AIDS education program, men and women who received a seven-session group HIV prevention intervention that focused on attitude, skill, and risk reduction behavior change strategies reported greater reductions across a range of sexual risk indicators. Because consistent condom use has been linked to reductions in HIV seroincidence (13-15) and because reductions in frequency of unprotected sex also predict lower levels of STD incidence, the behavioral effects of the intervention carry considerable public health importance.

The findings from this trial provide some direct evidence that the intervention also reduced disease outcomes. Relative to control condition members, participants who received the group intervention were less likely to report symptoms indicative of syndromal STDs through the follow-up period. Clinic chart data available for men and women recruited from STD clinics were used to identify new STDs in the follow-up period, incorporating clinician diagnosis, serology, and laboratory culture tests performed in the clinics. These data revealed similar overall rates of STD reinfection among intervention and control participants. However, for gonorrhea in men-an STD with high incidence in this population, which produces symptoms in men that usually result in treatment-seeking-lower incidence was observed among intervention condition participants compared with controls. Unexpectedly low point prevalence of gonorrhea and chlamydia at the 12-month follow-up limited the likelihood of observing between-group differences. However, the pattern of LCR findings on gonorrhea was consistent with the other STD symptom, diagnosis, and behavioral change data.

Because HIV infection may be acquired during unprotected intercourse and is a disease linked to behavior, change in sexual behavior practices is an appropriate outcome in HIV prevention studies. Of necessity, these practices must be measured through participants' self-reports of their sexual behavior. Previous research has established the validity and reliability of sexual risk behavior self-reports $(16,17)$. The current study sought to minimize threats to the reliability and validity of participants' reports of their sexual practices by pilot testing of questions to elicit sexual behavior data and training interviewers to elicit sexual practice reports in a nonjudgmental and unbiased manner. The consistency and magnitude of change found across multiple indicators of sexual risk behavior-including frequency of unprotected intercourse, levels of condom use, and patterns of consistent condom use-strengthen confidence in the behavior change outcomes produced by the intervention.

Two findings of the study warrant attention in future research. First, although reductions in sexual risk behavior in the intervention condition were significantly greater than those in the control condition, risk behavior also decreased among control condition members. A large portion of the participants were recruited from STD clinics, where they are counseled to reduce their risky behavior. Additionally, the process of self-evaluation, as would occur during in-depth assessment of risk behavior, may enhance motivation to change.

No past trials of group HIV preventive interventions have examined changes in both self-reported sexual risk practices and markers of subsequent disease incidence. The use of incident STDs as an indicator of the effectiveness of HIV prevention programs has often been advocated (18). STD symptom self-reports, shown in past research to correlate highly with laboratory tests for STDs (3), may serve as another indicator of disease associated with sexual behavior. Medical chart reviews-especially when supported by clinical evidence of disease and laboratory test findings, as in this study-yield additional data that complement self-reported sexual behavior. However, chart and symptom data are imperfect because many STDs are asymptomatic, especially among women, and not all patients with STDs seek treatment or are treated consistently at the same clinic. Although diagnostic procedures such as the LCR assay of urine can detect certain STDs with considerable sensitivity, they can detect only diseases present at the time of testing. Incident disease would not be detected in individuals who acquired and received treatment for an STD earlier in a follow-up period. For these reasons, research on the effectiveness of interventions to reduce sexual risk behavior should use a variety of outcome indicators.

Finally, several factors may have reduced the likelihood of observing between-group differences on biological outcome indicators. Notwithstanding the strict intake criteria and the known high risk behavior among STD clinic populations, observed rates of STDs were lower than anticipated. Only $75 \%$ of the medical charts of STD clinic participants were available for review, although chart recovery rates were the same across conditions. Some participants may have been treated for STDs at alternative clinical settings, further attenuating the amount of measurable disease. In sum, although imperfect, biological measures of
STDs are an important element of comprehensive evaluation of HIV prevention interventions because they are not subject to the limitations of self-reported sexual behavior.

Collectively, these findings indicate that the intervention was successful in reducing HIV-related sexual risks over a 1-year period. Nonetheless, it is equally important that strategies be developed to maintain longer term behavior changes in low-income, urban minority populations. Even if the reductions in HIV-related behavior observed in this study were maintained for only 1 year, however, they would still have a profound cost-effective public health impact in terms of the numbers of cases of HIV (and other STDs) averted in communities that adopted this intervention (19). These results lend support to the desirability of delivering behavioral, skill-based interventions in settings serving persons at high risk for HIV as one public health strategy to reduce HIV transmission.

\section{REFERENCES AND NOTES}

1. H. D. Gayle, Epidemiology of HIV infection and AIDS: National and international perspective, in Interventions to Prevent HIV Risk Behaviors: Programs and Abstracts of an NIH Consensus Development Conference (Office of the Director, National Institutes of Health, Bethesda, MD, 1997), pp. 23-29.

2. M. Fishbein and R. Coutinho, Eds., NIMH Multisite HIV Prevention Trial, AIDS 11 (suppl. 2) (December 1997).

3. J. N. Wasserheit, Sex. Transm. Dis. 19, 61 (1992).

4. D. A. Murphy, M. J. Rotheram-Borus, S. Srinivasa, W. K. Hunt, AIDS Behav., in press.

5. AIDS: Changing the Rules, a videotape available in English and Spanish, was used for the control intervention. Copyright: AIDSFilms. Distributed by Select Media, 60 Warren Street, 5th Floor, New York, NY 10007

6. P. J. Diggle, K.-Y. Liang, S. L. Zeger, Analysis of Longitudinal Data (Oxford Univ. Press, New York, 1994).

7. Community Intervention Trial for Smoking Cessation (COMMIT), Am. J. Public Health 85, 183 (1995).

8. Over $80 \%$ of both the intervention and control participants reported having been tested for HIV before the baseline assessment. About $43.5 \%$ of those tested did not report the result of the test either because they never returned for the result or because they refused to reveal the result. Of those reporting their HIV test result, 43 of 867 intervention participants and 53 of 861 control participants reported being HIV positive. Responses for the follow-up period do not cover the entire follow-up period as participants were only asked whether they had been tested during the 90 days before each interview, and, again, many participants refused to provide test results. Thus, reliable seroconversion data are not available.

9. For additional data, see www.sciencemag.org/ feature/data/976101.shl

10. T. R. Eng and W. T. Butler, Eds., The Hidden Epidemic: Confronting Sexually Transmitted Diseases (National Academy Press, Washington, DC, 1997).

11. E. W. Hook III and H. H. Handsfield, in Sexually Transmitted Disease, K. K. Holmes, P.-A. Mardh, P. F. Sparling, P. J. Wiesner, Eds. (McGraw-Hill, New York, ed. 2, 1990), pp. 149-165.

12. CDC (Centers for Disease Control and Prevention), Morb. Mortal. Wkly. Rep. 42 (no. RR-14), 56 (1993).

13. I. de Vincenzi, N. Engl. J. Med. 331, 341 (1994)

14. N. S. Padian, T. R. O'Brient, Y. Chang, S. Glass, D. P. Francis, J. Acquired Immune Defic. Syndr. 6 
1043 (1993)

15. D. D. Celentano et al., J. Am. Med. Assoc. 275, 122 (1996)

16. H. G. Miller, C. F. Turner, L. E. Moses, in AIDS: The Second Decade (National Academy Press, Washington, DC, 1990), pp. 359-472.

17. R. A. Coates et al., Am. J. Epidemiol. 128, 719 (1988)

18. Consensus Development Panel, Consensus Development Panel Report on HIV Prevention. (National Institutes of Health, Washington, DC, 1997).

19. D. R. Holtgrave et al., Public Health Rep. 110, 133 (1995)

20. The NIMH Multisite HIV Prevention Trial Group (listed in alphabetical order within each group).

Research Steering Committee [Site Principal Investigators (PI) and NIMH Staff Collaborator]: David D. Celentano, Johns Hopkins University, Baltimore, MD Colleen Dilorio, Emory University, Atlanta, GA; Tyler Hartwell, Research Triangle Institute, Research Triangle Park, NC; Jeffrey Kelly, Medical College of Wisconsin, Milwaukee; Raul Magana, University of California, Irvine; Edward Maibach, formerly PI at Emory University, Atlanta, GA Ann O'Leary, Rutgers University, New Brunswick, NJ; Willo Pequegnat, National Institute of Mental Health,

Rockville, MD; Mary Jane Rotheram-Borus, University of
California, Los Angeles; Robert Schilling, Columbia University, New York, NY.

Collaborating Scientists-Co-Principal Investigators: Jaime Amsel, formerly at University of California, Irvine; Nabila El-Bassel, Columbia University, New York, NY; Joao B. Ferreira-Pinto, formerly at University of California, Irvine; Alice Gleghorn, formerly at Johns Hopkins University, Baltimore, MD; Andre Ivanoff, Columbia University, New York; John Barton Jemmott III, Princeton University, Princeton, NJ; Loretta Sweet Jemmott, University of Pennsylvania, Philadelphia, PA Rebecca Martin, Johns Hopkins University, Baltimore, MD; Sutherland Miller, consultant, University of California, Los Angeles; Debra Murphy, University of California, Los Angeles; Marcella Raffaelli, formerly at Rutgers University, New Brunswick, NJ; Anne M. Rompalo, Johns Hopkins University, Baltimore, MD; William Schlenger, Research Triangle Institute, Research Triangle Park, NC; Kathleen Sikkema, Medical College of Wisconsin, Milwaukee; Anton Somlai, Medical College of Wisconsin, Milwaukee.

Site Managers: Kevin Alford, Johns Hopkins University, Baltimore, MD; Carlos Allende-Ramos, Rutgers University, New Brunswick, NJ; Kristin Hackl, Medical College of Wisconsin, Milwaukee; Mark Kuklinsky, University of California, Los Angeles; Esther Lopez, Univer- sity of California, Irvine; Patricia Nava, University of California, Irvine; Michelle Parra, University of California, Los Angeles; Jennifer Pranke, Rutgers University, New Brunswick, NJ; Helen Reid, University of California, Los Angeles; Jennifer Sharpe-Potter, Emory University, Atlanta, GA; Susan Witte, Columbia University, New York, NY; Yvette Villasenor, University of California, Irvine.

Data Coordinating Center: Nellie Hansen, Lisa LaVange, Deborah McFadden, Rebecca Perritt, W. Kenneth Poole, Research Triangle Institute, Research Triangle Park, NC.

Core Laboratory: Charlotte Gaydos and Thomas C. Quinn, Johns Hopkins School of Medicine, Baltimore, MD.

Research Support Office: Leonard Mitnick, Sherry Roberts, Ellen Stover, National Institute of Mental Health, Rockville, MD.

Data Safety and Monitoring Board: Alan S. Bellack, University of Maryland, College Park; Thomas Coates, University of California at San Francisco; William D. Crano, University of Arizona, Tucson; David Francis, University of Houston, Houston, TX; Sylvan B. Green, National Cancer Institute, Bethesda, MD; Karla Moras, University of Pennsylvania, Philadelphia, PA.

10 November 1997; accepted 9 April 1998

\section{So instant, you don't need water...}

\section{NEW! SCIENCE Online's Content Alert Service}

There's only one source for instant updates on breaking science news and research findings: SCIENCE's Content Alert Service. This free enhancement to your SCIENCE Online subscription delivers e-mail summaries of the latest research articles published each Friday in SCIENCE - instantly. To sign up for the Content Alert service, go to SCIENCE Online - and save the water for your coffee.

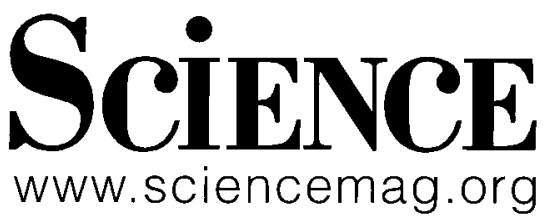

For more information about Content Alerts go to www. sciencemag.org.

Click on Subscription button, then click on Content Alert button. 


\section{Science}

The NIMH Multisite HIV Prevention Trial: Reducing HIV Sexual Risk Behavior

The National Institute of Mental Health (NIMH) Multisite HIV Prevention Trial Group

Science 280 (5371), 1889-1894.

DOI: $10.1126 /$ science.280.5371.1889

ARTICLE TOOLS

http://science.sciencemag.org/content/280/5371/1889

REFERENCES

This article cites 6 articles, 0 of which you can access for free http://science.sciencemag.org/content/280/5371/1889\#BIBL

PERMISSIONS

http://www.sciencemag.org/help/reprints-and-permissions 